\title{
The H I-rich Elliptical Galaxy NGC 5266: An Old Merger Remnant?
}

\author{
R. Morganti ${ }^{1}$, E. M. Sadler ${ }^{2}$, \\ T. A. Oosterloo ${ }^{3}$, A. Pizzella ${ }^{4}$ and F. Bertola ${ }^{4}$ \\ ${ }^{1}$ IRA, CNR, via Gobetti 101, 40129 Bologna, Italy, \\ and ATNF, CSIRO, PO Box 76, Epping, NSW 2121, Australia \\ rmorgant@atnf.csiro.au \\ ${ }^{2}$ School of Physics, University of Sydney, NSW 2006, Australia \\ ${ }^{3}$ ATNF, CSIRO, PO Box 76, Epping, NSW 2121, Australia \\ ${ }^{4}$ Dipartimento di Astronomia, Vicolo dell'Osservatorio 5, 35122 Padova, Italy
}

Received 1996 August 5, accepted 1996 November 12

\begin{abstract}
We present $\mathrm{H}_{\mathrm{I}}$ images for the dust-lane elliptical galaxy NGC 5266. This galaxy contains more than $10^{10} M_{\odot}$ of $\mathrm{HI}$ and our data show that the neutral hydrogen extends to $\sim 8$ arcmin each side of the nucleus, or eight times the optical half-light radius $R_{\mathrm{e}}$. Surprisingly, the outer $\mathrm{H}_{\mathrm{I}}$ gas extends almost orthogonally to the optical dust lane. The overall $\mathrm{H}_{\mathrm{I}}$ kinematics can be successfully modelled by assuming that the gas lies in two orthogonal planes-in the plane of the dust lane in the central parts and perpendicular to this in the outer regions. The large amount of neutral gas observed and the Hi morphology suggest that this object may have formed from the merger of two gas rich spiral galaxies. If so, it probably represents a relatively old merger remnant since most of the $\mathrm{H}_{\mathrm{I}}$ appears settled. The mass-to-light ratio has also been derived, with evidence for a dark matter halo around this galaxy.
\end{abstract}

Keywords: galaxies: individual (NGC 5266) — galaxies: kinematics and dynamics

\section{Introduction}

NGC 5266 is a bright E4 elliptical galaxy $\left(V_{\mathrm{h}}=\right.$ $3044 \mathrm{~km} \mathrm{~s}^{-1}$ ) with a prominent dust lane along its projected minor axis. It has been studied in detail optically (Caldwell 1984; Varnas et al. 1987), where it shows that the stars rotate about the optical minor axis while the gas in the dust lane rotates about the optical major axis, i.e. the kinematic axes of the stars and gas appear to be orthogonal. Both Caldwell (1984) and Varnas et al. (1987) suggested that the underlying galaxy is triaxial.

From narrowband imaging in the $\mathrm{H} \alpha$ and [N II] lines, Goudfrooij et al. (1994) showed that the ionised gas in NGC 5266 lies in a ring clearly associated with the dust ring. Sage \& Galletta (1993) made CO observations of NGC 5266 and found that the molecular gas also has a ring-like distribution and is co-rotating with the ionised gas. Varnas et al. (1987) also detected $\mathrm{H}_{\mathrm{I}}$ in NGC 5266 using the Parkes $64 \mathrm{~m}$ radio telescope and found that the galaxy contains more than $10^{10} M_{\odot}$ of neutral hydrogen, one of the largest amounts observed in any elliptical galaxy.

Here, we present the results obtained from $\mathrm{HI}_{\mathrm{I}}$ observations of this galaxy done with the Australia Telescope Compact Array (ATCA). Preliminary results were presented in Morganti et al. (1995), while more details and a complete discussion of the ATCA observations are given in Morganti et al. (1996).

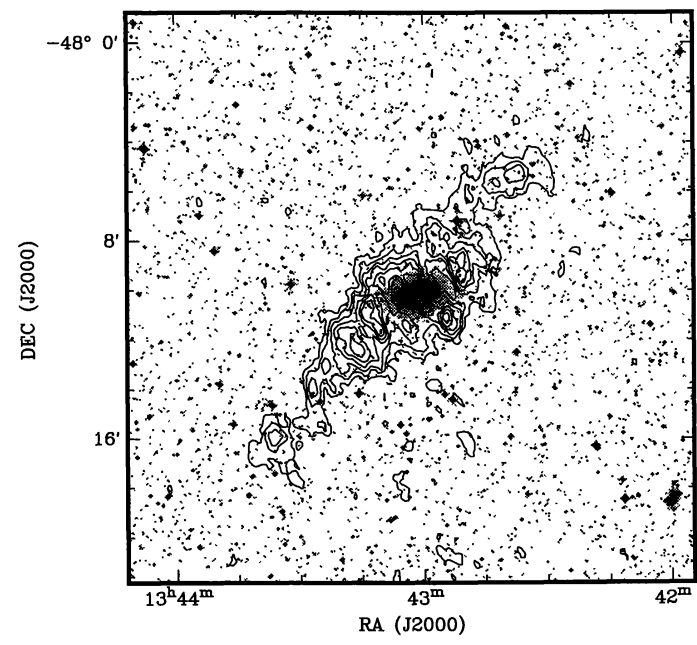

Figure 1-Map of the total $\mathrm{HI}$ distribution (contours) superimposed on the optical image from the Sky Survey.

2 Distribution and Kinematics of $H_{I}$ in NGC 5266

We have observed NGC5266 with four different ATCA configurations $(375 \mathrm{~m}$ and three $1.5 \mathrm{~km}$ configurations) and we detect a total $\mathrm{HI}$ mass of $2.4 \times 10^{10} M_{\odot}$, which agrees with the Parkes single-dish measurements made by Varnas et al. (1987).

$1323-3580 / 97 / 010089 \$ 05.00$ 
The total Hi map is shown in Figure 1 superimposed on the optical image. It is clear that the neutral hydrogen in NGC 5266 is very extended. The total extent is about 16 arcmin, i.e. about $\sim 140 \mathrm{kpc}$ corresponding to $\sim 8$ times the optical half-light radius $R_{\mathrm{e}}$ on each side. The most striking feature is that the $\mathrm{HI}$ is elongated along p.a. 135 , i.e. nearly perpendicular to the orientation of the dust lane (p.a. $\sim 21^{\circ}$ ). Although in most dust-lane and polar-ring galaxies where $\mathrm{H}_{\mathrm{I}}$ is been observed, it lies in the same plane as the dust lane or ring, the presence of an outer $\mathrm{H}_{\mathrm{I}}$ ring perpendicular to the inner dust lane has been observed before in a few cases (NGC 2685, Peletier \& Christodoulou 1993; NGC 5128, Schiminovich et al. 1994). From the position-velocity map at p.a. $=135^{\circ}$ (i.e. the major axis, Figure 2) it is possible to see that there are actually signatures of three distinct structures. The first is a central, fast-rotating component associated with the dust lane. In the position-velocity plot along the minor axis (not shown here) this fastrotating component appears to be spatially resolved, with the western side of this structure redshifted compared to the systemic velocity, i.e. it rotates in the same sense as the ionised gas and CO. The second structure is the main $\mathrm{H}_{\mathrm{I}}$ component, which can be traced out to 4 arcmin radius. It has a quite regular velocity field, implying that this component is a rotating annulus roughly perpendicular to the dust lane. Interestingly, a high-contrast optical image of NGC 5266 from D. Malin (Figure 3) shows that there is a good correspondence between the faintest optical isophotes and the main (ellipticalshaped) neutral gas structure, implying that it has a faint optical counterpart. The third component corresponds to the two outermost arm structures which could represent either an edge-on ring or two tidal arms.

Thus the data suggest that the neutral hydrogen inside 4 arcmin lies in two orthogonal planes. This has been confirmed by a simple modelling of the $\mathrm{HI}$ kinematics where we have assumed that the neutral gas inside 1 arcmin is coincident with the dust lane, but that somewhere further out it shifts to a perpendicular plane. The position angle of this outer plane happens to be very close to the position angle of the stellar major axis (Caldwell 1984; Varnas et al. 1987), consistent with the idea that the outer $\mathrm{H}$ I distribution has settled in a symmetry plane of the stellar galaxy. The transition from one plane to the other lies just beyond the optical dust lane and is quite abrupt, though there is evidence for a small amount of gas 'connecting' the outer region of the optical dust band and the inner part of the main $\mathrm{H}_{\mathrm{I}}$ disk.

If we assume the rotation velocity to be constant with radius, we find the best fit to be for $V_{\text {rot }}=270 \mathrm{~km} \mathrm{~s}^{-1}$, which is close to the values

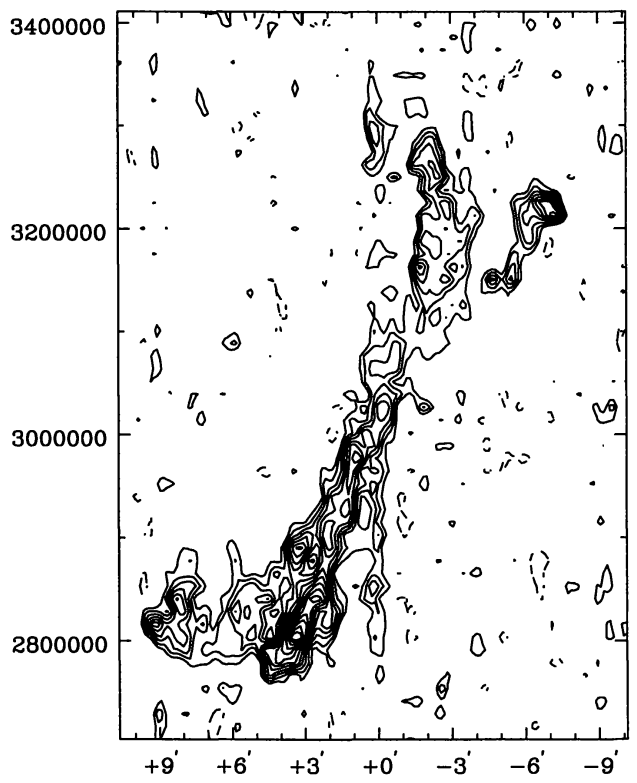

Figure 2-Position-velocity map obtained from a slice in p.a. $=135^{\circ}$ and width 90 arcsec. The contour levels are $-0.011,0.011$ to 0.08 in steps of $0.006 \mathrm{mJy} /$ beam. The velocity is in units of $\mathrm{ms}^{-1}$.

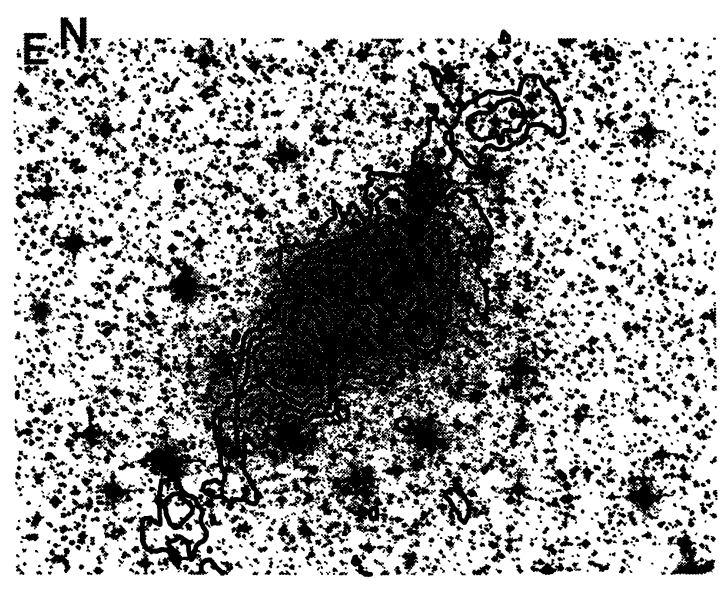

Figure 3-Total H I (contours) superimposed on a deep optical image (grey).

determined optically and from $\mathrm{CO}$ observations. Our modelling is also telling us that the rotation velocity cannot be much lower than $250 \mathrm{~km} \mathrm{~s}^{-1}$.

The $\mathrm{HI}$ emission from the outermost regions ( $>4$ arcmin) cannot be reproduced by our simple model, suggesting that the outer parts might be tidal tails of neutral gas formed in a merger, and therefore very unrelaxed structures. Alternatively, they could be part of an outer, edge-on disk or ring, as some characteristics of their kinematics seem to suggest.

Assuming a constant rotation velocity of 270 $\mathrm{km} \mathrm{s}^{-1}$ and a spheroidal mass distribution with an 
intrinsic flattening of $q=0.6$ (as indicated by the optical image of the galaxy), we can derive the mass of the galaxy. Adopting an $r^{\frac{1}{4}}$-law light profile with $r_{\mathrm{e}}=65^{\prime \prime}$ (Varnas et al. 1987), we find that the derived mass-to-light ratio ranges from $M / L_{\mathrm{B}}$ $\sim 4$ at 1 arcmin radius to $M / L_{\mathrm{B}}=8$ at 4 arcmin. Comparing our derived values with that measured for the central $M / L_{\mathrm{B}}$ from optical observations $\left(M / L_{\mathrm{B}}=3.0\right.$ at $0.3 R_{\mathrm{e}}$, Bertola et al. 1993), we find that $M / L_{\mathrm{B}}$ increases by a factor $\sim 2 \cdot 7$ between the centre and $r=4 R_{\mathrm{e}}$. If we use the outermost $\mathrm{HI}$ to estimate the mass-to-light ratio beyond 4 arcmin radius, we derive $M / L_{\mathrm{B}} \sim 16$ within 8 arcmin radius. However, as discussed earlier, it is likely that the two outer $\mathrm{H}$ I structures are not in equilibrium.

\section{Origin of the Gas Disk in NGC 5266}

The two orthogonal gas distributions in NGC 5266 have probably settled into two stable planes: this is characteristic of a triaxial galaxy with a stationary potential (Heiligman \& Schwarzschild 1979). However, although most of the $\mathrm{HI}_{\mathrm{I}}$ lies in these two planes, there are some hints in the data that the two disks might be part of a single warped structure. If so, NGC 5266 would resemble some of the classical warps, such as NGC 3718 (Schwarz 1985) or NGC 660 (Gottesman \& Mahon 1990; Arnaboldi \& Galletta 1993), though with the important difference that relatively little gas is found at intermediate radii. Possibly this means that the warp is 'breaking up' into two disks. Perhaps the warp is due to slow figure rotation, which would have to be retrograde, as suggested by Varnas et al. (1987).

A puzzling feature of NGC 5266 is the origin of the huge amount of $\mathrm{HI}$. It is generally believed that the $\mathrm{HI}_{\mathrm{I}}$ in elliptical galaxies has an external origin (e.g. Knapp, Turner \& Cunliffe 1985). If NGC 5266 is the result of a merger, it seems likely that two gas-rich spiral galaxies must have been involved in order to provide the large $\mathrm{HI}_{\mathrm{I}}$ mass that we observe. Furthermore, a large amount of neutral gas must have survived the collision without forming stars. NGC 5266 shows, indeed, a ratio of the total hydrogen mass to the total (blue) luminosity $\left(M_{\mathrm{HI}} / L_{\mathrm{B}} \sim 0 \cdot 2\right)$ which is a typical value for spiral galaxies but much higher than those seen in most ellipticals.

The numerical simulations for a merger of two spirals presented by Hibbard \& Mihos (1995) for the case of NGC 7252 show that a relatively old merger $(t>1.2 \mathrm{Gyr})$ can have a similar gas morphology to that observed in NGC 5266, i.e. part of the $\mathrm{H}_{\mathrm{I}}$ is settling in the galaxy while the tidal arms are also still present. Thus it seems likely that NGC 5266 is a similar system to NGC 7252 , but at a later stage in its evolution.

If this is the case, NGC 5266 would have already passed the period of high activity and rapid star formation that is believed to result from the merger of two spirals. This is supported also by its IR characteristics. The very low rate of current star formation in this object (despite the large mass of $\mathrm{HI}_{\mathrm{I}}$ could be due to the low surface density of $\mathrm{HI}$ gas, which cannot support star formation (Kennicutt 1989).

\section{Conclusions}

We have presented $\mathrm{H}$ I images of the gas-rich elliptical galaxy NGC5266. We find that the gas in this galaxy lies in two orthogonal planes; some $\mathrm{HI}_{\mathrm{I}}$ is associated with an inner, minor-axis dust lane but the majority lies in an extended, outer distribution aligned with the stellar major axis. Using this outer component as a mass tracer, we find a mass to-light ratio $M / L_{\mathrm{B}} \sim 8$ within $4 R_{\mathrm{e}}$, which is similar to that derived for other ellipticals at similar radii and suggests the presence of dark matter. The most likely origin for this gas in NGC 5266 appears to be the merger of two gas-rich spirals, and the settled nature of the gas suggests that this probably occurred at least $1 \cdot 2 \mathrm{Gyr}$ ago.

Arnaboldi, M., \& Galletta, G. 1993, A\&A, 268, 411

Bertola, F., Pizzella, A., Persic, M., \& Salucci, P. 1993, ApJ, 416, L45

Caldwell, N. 1984, ApJ, 278, 96

Goudfrooij, P., De Jong, T., Hansen, L., \& Norgaard-Nielsen, H. U. 1994, MNRAS, 271, 833

Gottesman, S. T., \& Mahon, M. E. 1990, in Paired and Interacting Galaxies, ed. J. W. Sulentic, W. C. Keel \& C. M. Telesco (Washington DC: NASA), 209

Heiligman, G., \& Schwarzschild, M. 1979, ApJ, 233, 872

Hibbard, J. E., \& Mihos, J. C. 1995, AJ, 110, 140

Kennicutt, R. C. 1989, ApJ, 344, 685

Knapp, G. R., Turner, E. L., \& Cunniffe, P. E. 1985, AJ, 90,454

Morganti, R., Pizzella, A., Sadler, E. M., \& Bertola, F. 1995, PASA, 12, 143

Morganti, R., Sadler, E. M., Oosterloo, T., Pizzella, A., Bertola, F., \& Pizzella A. 1997, AJ, in press

Peletier, R. F., \& Christodoulou, D. M. 1993, AJ, 105, 1378

Sage, L., \& Galletta, G. 1993, ApJ, 419, 544

Schiminovich, D., van Gorkom, J. H., van der Hulst, J. M., \& Kasow, S. 1994, ApJ, 423, L101

Schwarz, U. 1985 A\&A, 142, 273

Varnas, S. R., Bertola, F., Galletta, G., Freeman, K. C., \& Carter, D. 1987, ApJ, 313, 69 